\title{
AGN populations in compact groups of galaxies
}

\author{
A. S. Amirkhanian ${ }^{1}$, A. del Olmo ${ }^{2}$, A. G. Egikian ${ }^{1}$,

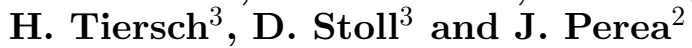 \\ ${ }^{1}$ Byurakan Astrophysical Observatory, Aragatzotn Province, Armenia \\ ${ }^{2}$ Instituto de Astrofisica de Andalucia, Granada, Spain \\ ${ }^{3}$ Sternwarte Koenigsleiten, Muenchen, Germany
}

\begin{abstract}
Compact groups of galaxies (CGG) have revealed some interesting problems from their origin and lifetime to the evolution of their members in such dense configurations. Some authors suppose that CGG probably are the best location for AGNs in the local Universe. According to our preliminary data about $7-10 \%$ of member galaxies in Shahbazian compact groups (SHCGs) are emission-line galaxies including the broad-line AGN and the narrow emission-line galaxies. Shahbazian 355/4 is a classical Seyfert 1 galaxy at the same redshift as host group. Moreover Shahbazian 278/4 is also a broad-line AGN in an early-type galaxy. This is the first emission-line object in SHCGs. Meanwhile there is no Seyfert 1 galaxy among the spectroscopically investigated galaxies in the South compact groups, although more than $70 \%$ of the member galaxies in these groups probably have an active nucleus. The UZC- compact groups have an excess of Seyfert 2s (but not Seyfert 1s!). Further observational studies are necessary to understand such and many other questions related to the puzzle of CGG.
\end{abstract}

Keywords. Shahbazian groups, AGN, Starburst galaxies

Compact groups of galaxies have revealed a number of interesting problems from their origin and lifetime to the evolution of their members in such dense configurations (Hickson (1997) and references therein). Some authors suppose that compact groups of galaxies probably are the best location for AGNs in the local Universe (Coziol et al. (2000), Shimada et al. 2000). Hickson 31 (Arp 259) is one of the most remarkable compact groups studied till now with two member Markarian galaxies (Rubin et al. (1990). HCG 31 (G) is Mrk 1089 with a known Wolf-Rayet (WR) spectrum. HCG 31 (G) is Mrk 1090. This is a starburst galaxy. The redshift of this group is $\mathrm{z}=0.0131$. IRAS $23532+2513$ with a redshift of 0.056 is an example of a compact group including a Seyfert 1 and a starburst galaxy (Zhen-Long Zou et al. (1995).

According to our preliminary data about $7-10 \%$ of member galaxies in Shahbazian compact groups are the emission line galaxies including the broad-line AGNs (of classical Seyfert 1 type) and the narrow emission-line galaxies (Tiersch et al. (1999), Amirkhanian et al. (2010). Their redshift makes these galaxies a physical member of the hosted group. Shahbazian 355/4 is a classical Seyfert 1 galaxy (Fig. 1). (discovered by our research group) at the same redshift $(\mathrm{z}=0.0942)$ as hosted group. Moreover Shahbazian 278/4 is also the broad-line AGN in an early-type galaxy. This is the first emission-line object in Shahbazian compact groups discovered by Spanish astronomers (del Olmo and Moles (1991). At the same time there is no Seyfert 1 galaxy among the spectroscopically investigated galaxies in the South compact groups, although more than $70 \%$ of the member galaxies in these groups probably have an active nucleus (Coziol et al. (2000). Why? The UZC- compact groups have an excess of Seyfert 2s (but not Seyfert 1s!) (Kelm et al. (2004). Why? Further observational studies are necessary to understand such and many 


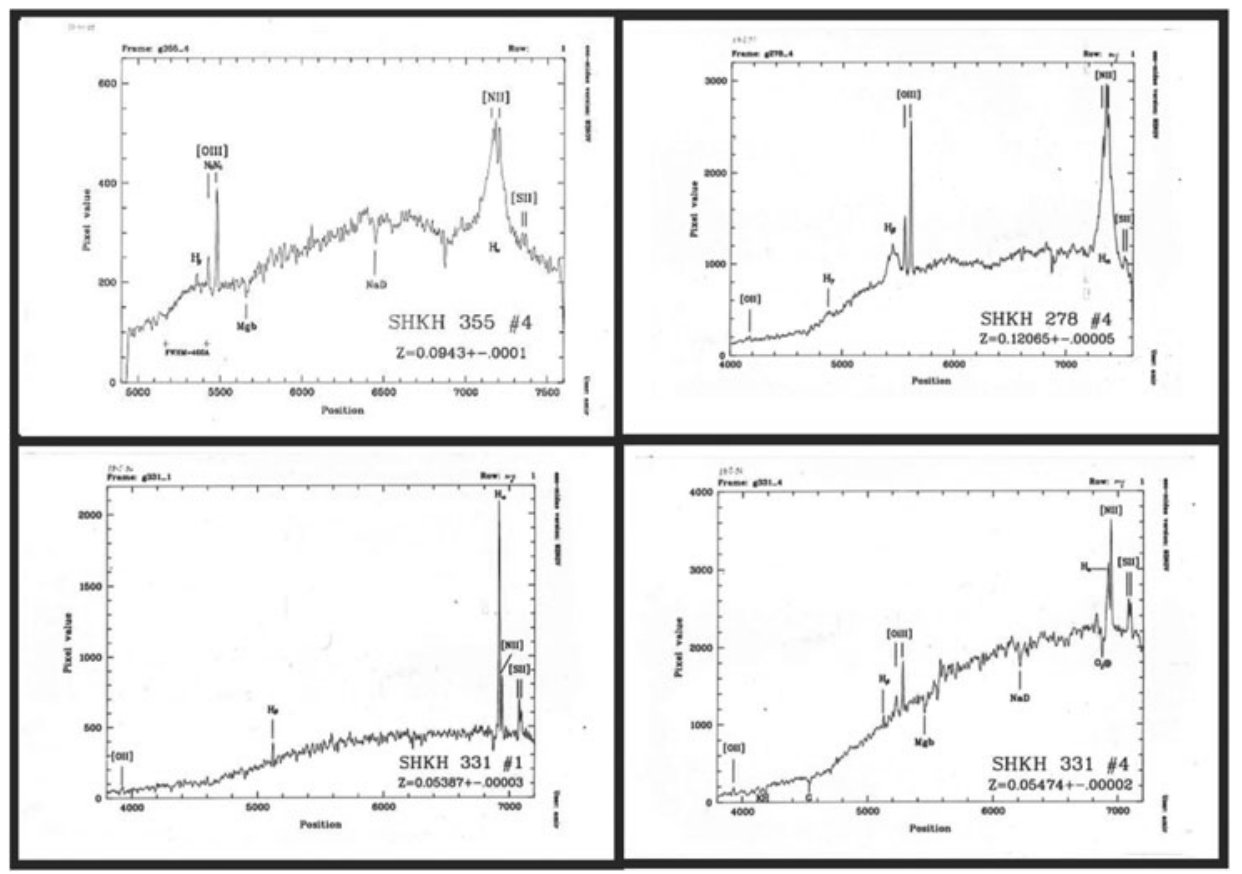

Figure 1. The spectra of some Emission-Line Galaxies (ELGs) in SHCGs. All except one were taken with the $1.5 \mathrm{~m}$ Danish telescope at La Silla (Chile). The spectrum of Seyfert 1 galaxy Sh $355 / 4$ was obtained using the $2.2 \mathrm{~m}$ telescope at Calar Alto (Almeria, Spain). Original dispersion is 2.9 A per pixel, integration 3600 s.

other questions related with the puzzle of compact groups.

Acknowledgements. This work was supported by a grant of the "Deutsche Forschungsgemeinschaft" (for ASA and AGE). This project was also supported by a grant of the NATO Scientific Committee (for ASA) and a grant of the Junta de Andalucia (for AGE). We (ASA and AGE) would like also to thank the administration and personnel of the IAA for their welcome and hospitality.

\section{References}

Amirkhanian, A. S., Egikian, A. G., del Olmo A., \& Perea, J. 2010, in: H. Harutyunian, A. Mickaelian and Y. Terzian, (eds.), Evolution of Cosmic Objects through their Physical Activity, Proc. of the Conf. dedicated to V. A. Ambartsumian's 100th anniversary, Yerevan, "Gitutyun" Publ. House, p. 225

Coziol, R., Iovona, A., \& de Carhvalo, R. R. 2000, AJ 120, 47

del Olmo, A. \& Moles, H. 1991, A\&SA 245, 27

Hickson, P. 1997, ARAA 35, 357

Kelm, B., Focardi, P. \& Zitelli V. 2004, A\&SA 418, 25

Rubin, V. C., Hunter, D. A., \& Kent Ford, W. Jr. 1990, ApJ 365, 86

Shimada, M. et al. 2000, AJ 119, 2664

Tiersch, H., Stoll, D., Neizvestny, S., Amirkhanian, A. S., \& Egikian, A. G. 1999, in: Y. Terzian, D. Weedman \& E. Khachikian, \& (eds.), Active Galactic Nuclei and Related Phenomena, Proc. IAU Symposium No. 194 (Chelsea, Michigan: ASP), p. 394

Zhen-Long Zou, et al. 1995, A\&A 304, 369 